\title{
Numerical Simulations Study of a Spin-1 Blume-Emery-Griffiths Model on a Square Lattice
}

\author{
L. Luque, ${ }^{1}$ S. A. Grigera, ${ }^{1,2}$ and E. V. Albano ${ }^{1}$ \\ ${ }^{1}$ Instituto de Física de Líquidos y Sistemas Biológicos, UNLP-CONICET, La Plata 1900, Argentina \\ ${ }^{2}$ SUPA, School of Physics and Astronomy, University of St Andrews, North Haugh, St Andrews KY16 9SS, UK
}

(Dated: April 5, 2019)

\begin{abstract}
We use the Monte Carlo simulation technique to study the critical behavior of a three-state spin model, with bilinear and biquadratic nearest-neighbor interactions, known as the Blume-EmeryGriffiths model (BEG), in a square lattice. In order to characterize this model, we study the phase diagram, in which we identify three different phases: ferromagnetic, paramagnetic and quadrupolar, the later with one sublattice filled with spins and the other with vacancies. We perform our studies by using two algorithms: Metropolis Update (MU) and Wang-Landau (WL). The critical scaling behavior of the model is complementary studied by applying results obtained by using both algorithms, while tricritical points and the tricritical scaling behavior is analyzed by means of WL measuring the joint density of states and using the method of field mixing in conjunction with finite-size scaling. Furthermore, motivated by the decoupling between spins observed within the quadrupolar phase, we further generalize the BEG model in order to study the behavior of the system by adding a next nearest neighbour (NNN) interaction between spins. We found that by increasing the strength of the (ferromagnetic) NNN interaction, a new ferromagnetic phase takes over that contains both quadrupolar and ferromagnetic order.
\end{abstract}

\section{INTRODUCTION}

The Blume-Emery-Griffiths (BEG) model $^{1}$ is an effective spin-lattice model that was proposed in 1971 for the study of some features of the thermodynamic behavior of $\mathrm{He}^{3}-\mathrm{He}^{4}$ mixtures, such as phase separation and superfluidity. At each site there is a spin that can take values \pm 1 and 0 , considered as a vacancy. It is based on the Blume-Capel model ${ }^{2,3}$. Like its predecessor, it contains an Ising-like term for interaction between spins, and one that controls the abundance of vacancies. In addition, the BEG incorporates interactions between vacancies. Since the introduction of the BEG model, many work has been devoted to its study and its applications in related fields such as ternary fluids ${ }^{4,5}$, phase changes in microemulsions ${ }^{6}$, semiconductor alloys ${ }^{7}$, electric conduction models ${ }^{8}$, etc. Also different techniques and methods have been used in order to understand the critical behavior as well as many interesting features of the model. This includes mean-field approximations ${ }^{1}$, high temperature series expansions ${ }^{9}$, Monte Carlo simulations ${ }^{10}$, renormalization group ${ }^{11-13}$, cluster variation methods ${ }^{14}$, effective field theories ${ }^{15-17}$, etc. Most of these techniques have also been used to characterize the $\mathrm{BC}$ model ${ }^{18,19}$, but in particular we recall studies of this model performed by using the Wang-Landau (WL) algorithm ${ }^{20,21}$. In fact, since both the BC and the BEG models exhibits tricritical behaviour, the WL can be used to determine the limiting probability distribution function of the scaling fields and their conjugate variables, which further allows to evaluate tricritical points and carry out tricritical scaling analysis ${ }^{20,22-26}$. Within this context, the aim of this paper is to determine the full phase diagram of the BEG model by performing complementary numerical simulations based on standard Metropolis update (MU) and the WL algorithm in the implementation pro- posed by Belardinelli-Pereyra ${ }^{27}$. Our analysis shows the great advantage of the use of complementary methods, while standard simulations allows us to study large sample sizes, the WL method grants unrestricted access to the values of temperatures and crystal fields, allowing the characterization of the tricritical behavior and its corresponding scaling. In this way, we identify three different phases: ferromagnetic $(F)$, paramagnetic $(P)$, and quadrupolar $(Q)$; as well as the tricritical line at the boundary between $F P$ phases. Furthermore, we generalize the BEG model in order to include NNN (ferromagnetic) interactions, finding the conditions for the appearance of an additional phase, $Q_{F}$, that contains both ferromagnetic and quadrupolar order.

We considered that the knowledge and understanding of the phase diagram of the BEG model, as discussed in the present paper, is essential for undertaking further studies. In particular we aim to characterize the dependence of quadratic interaction parameter (that regulates the interaction between vacancies) on the interfacial properties of the model, e.g. interfacial stiffness, surface tension, wetting behavior, etc., on view of the well known phenomena of 'interfacial segregation of vacancies" already observed in the BC model ${ }^{28-33}$.

\section{MODEL AND METHODS}

The Hamiltonian of the BEG model is given by:

$$
\mathcal{H}_{\mathrm{BEG}}=-J \sum_{\langle i, j\rangle} s_{i} s_{j}+D \sum_{i=1}^{N} s_{i}^{2}-\alpha J \sum_{\langle i, j\rangle} s_{i}^{2} s_{j}^{2},
$$

where the spin variable $s_{i}$ can take the values 1,0 and -1 , $J$ denotes the coupling constant, $D$ is the crystal field, $\alpha$ is a parameter characterizing the quadratic interaction, 
$\langle i, j\rangle$ indicates sum over nearest neighbors, and $N$ is the total number of sites. The results shown in this work are calculated using ferromagnetic coupling $(J>0)$, but can be straightforwardly mapped to the antiferromagnetic case by the usual transformation that reverses the sign of the spin variables of one of the disjoint sublattices of the square lattice.

One possible interpretation of this Hamiltonian sees the origin of the third term as coming from interaction between neighbouring vacancies $\left(s_{i}=0\right)$. In this scenario it is to be expected that $\alpha$ may influence the interfacial properties of the BEG model, for example by altering the surface tension. The BEG model has been studied extensively for the case of positive quadratic interactions $(\alpha>0)$, i.e. attractive interaction between vacancies, and its phase diagram is well understood ${ }^{34}$, but lately, the attention has been drawn to the understanding of the influence of the repulsive biquadratic term on the phase diagram $(\alpha<0)$, i.e. repulsive interaction between vacancies, which can lead to the occurrence of the socalled quadrupolar phase.

This additional type of ordered state takes place for suitable values of $D$ and (relatively) large negative values of $\alpha$. It consists, (at $T=0$ ), of a checkerboard arrangement of vacancies and occupied sites in the two disjoint sublattices of the square lattice. The spins of the occupied sites have no mutual interactions and thus the state is perfectly paramagnetic at all temperatures. The phase can be accurately described by means of an order parameter:

$$
Q=\frac{2}{N}\left(\sum_{f \in A} s_{f}^{2}-\sum_{g \in B} s_{g}^{2}\right),
$$

where $N$ is the total number of sites of the lattice and the sums over $A$ and $B$ correspond to the sites of the two sublattices.

In order to study the phase diagram of the BEG model we have performed numerical simulations ${ }^{35}$ using two different algorithms: the usual Monte Carlo Metropolis update $^{36}$ and Wang-Landau method for the evaluation of the density of states ${ }^{37,38}$ in the implementation proposed by Belardinelli-Pereyra ${ }^{27}$. In both types of simulations, the BEG model is mainly studied on a square lattice of dimension $L \times L$, with $L=16$, and assuming periodic boundary conditions, however the Metropolis algorithm is also applied to samples of different size up to $L=512$. A detailed description of the simulation methods can be found in the appendix.

\section{RESULTS}

\section{A. The $T=0$ phase diagram}

It is straightforward to calculate the $D-\alpha$ phase diagram of the BEG model at $T=0$ from simple energetic $\operatorname{arguments}^{39}$ (see the black dashed-lines in Fig. 1). The variable $D$ controls the number of vacancies present in the system: in the limit $D \rightarrow+\infty$ the system fills with vacancies, while in the limit $D \rightarrow-\infty$ the opposite is true and the system tends to the Ising model.

For $\alpha \geq-1$, there is a single ordered state: a ferromagnetic phase. A sufficient number of vacancies can lead into a disordered paramagnetic state along the line given by

$$
D=2 J(\alpha+1) .
$$

Note that for the particular case of $\alpha=0$, one recovers the well known Blume-Capel model with a single zero temperature critical point at $D=2 J$.

For $\alpha \leq-1$ there is a strong repulsion between neighbouring vacancies so that as a strongly negative $D$ is decreased in intensity and vacancies start to appear, they tend to fill one of the disjoint sublattices of the square lattice; this is the quadrupolar phase that takes place along the line

$$
D=4 J(\alpha+1) .
$$

This phase is also destabilised by the proliferation of vacancies, so that even at $T=0$ the system eventually disorders into a paramagnetic state above $D=0$.

At $T=0$ and $D=0$, the three phases coexist at a single critical point at $\alpha=-1$.

\section{B. Numerical Simulations}

Figure 1 was obtained by using the W-L algorithm and summarizes the main results of this work. The phase diagram as function of $D, \alpha$ and $T$ shows the existence of three different phases: a disordered paramagnet $(\mathrm{P})$, a ferromagnet $(\mathrm{F})$ and the quadrupolar phase $(\mathrm{Q})$. The transition between the paramagnetic and ferromagnetic phases shows a line of tricritical points (marked as black stars) separating a surface of second order (full line) and first order (dotted line) transitions. This tricritical line ends at $T=0$ at the point where the three phases coexist. The transition into the quadrupolar phase is always second order.

The existence of the ferromagnetic phase is easily determined by the value of the magnetization, $m=$ $\frac{1}{N} \sum_{i=1}^{N} s_{i}$ and its associated susceptibility, $\chi$. The transition has also signatures in the specific heat, but they are often overshadowed by the Schottky anomaly associated with the anisotropy of the system. The upper panel of Fig. 2 shows an example of the behaviour of the magnetization as a function of temperature, for $D=3$ and $\alpha=1$, when a second order phase transition takes place. The different symbols correspond to different lattice sizes. It can be seen that as the temperature is lowered there is a clear rise in the magnetization, that becomes increasingly sharp as the sample size is increased. The inset shows the same data plotted in terms of the reduced variables 


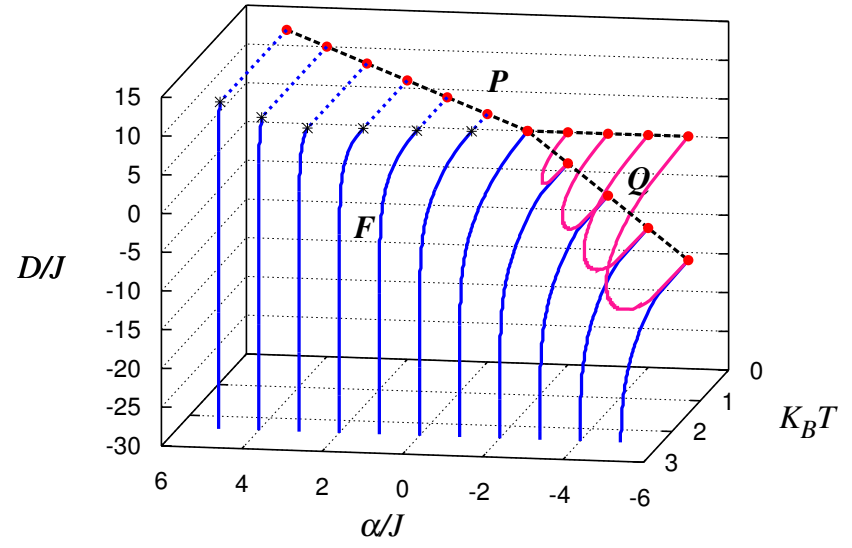

FIG. 1. Phase Diagram of the BEG Model. It shows the three phases: ferromagnetic, paramagnetic (blue) and quadrupolar (pink), separated by transition lines. The ferromagnetic $\leftrightarrow$ paramagnetic transition lines present first (dashed line) and second (complete line) order transitions, separated by tricritical points (stars). It also shows the coexistence line of the ground state (black dashed line) and the ground state for each value of $\alpha$ (red points). Figure obtained by using W-L algorithm.

$M L^{\frac{\beta}{\nu}}$ and $\left|T-T_{c}^{\infty}\right| L^{\frac{1}{\nu}}$ with $\nu=1$ and $\beta=0.125$. The collapse of these data is used to determine the value of $T_{c}^{\infty}=1.748$ and shows that the universality class of the transition is 2D Ising, well in agreement with previous studies of this model $^{10}$. This figure was built by using the MU algorithm, in order to compare the results obtained with W-L algorithm with the finite-size study of the system.

As we have mentioned, the ferromagnetic line changes from second to first order at a tricritical point. To determine the location of the second order critical temperature in the thermodynamic limit, one can make use of the fourth-order cumulant

$$
U=1-\frac{\left\langle m^{4}\right\rangle}{3\left\langle m^{2}\right\rangle^{2}},
$$

which should be scale invariant at the critical temperature, and hence show a size-independent crossing point. The scaling ansatz for the cumulant lacks of any size dependent prefactor, so

$$
U(L) \sim \tilde{U}\left(\left(T-T_{c}^{\infty}\right) L^{\frac{1}{\nu}}\right),
$$

where $\tilde{U}$ is a scaling function that does not need to be specified here. Then, the common intersection point of curves corresponding to samples of different sizes allow us to estimate the critical temperature. The lower panel of Fig. 2 shows the cumulant calculated for the same values of $D$ and $\alpha$. The second order critical temperature in this case is $T_{c}^{\infty}=1.748$.

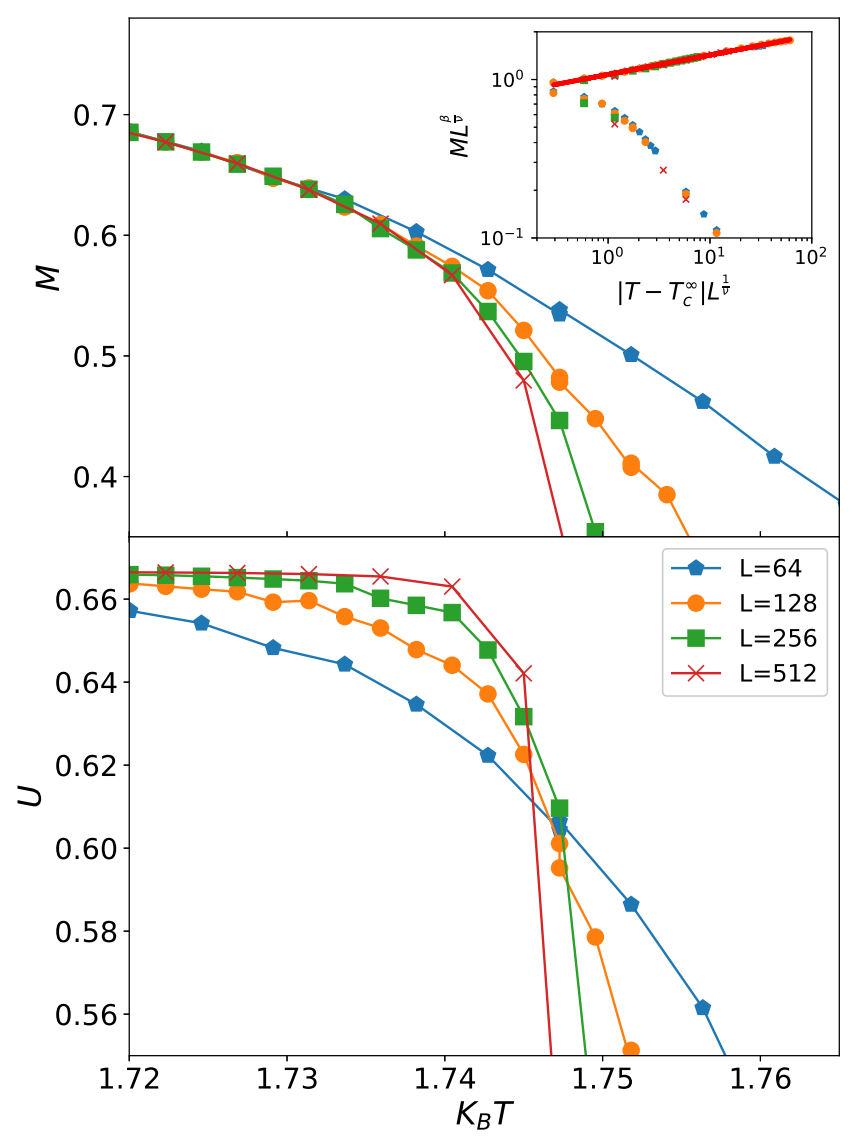

FIG. 2. Upper panel: plot of the Magnetization $\langle|m|\rangle$ vs Temperature. The sizes of the lattice are shown, and the parameters are $\alpha=1$ and $D=3$. Inset: Scaling plot of the $M L^{\frac{\beta}{\nu}}$ vs $\left|T-T_{c}^{\infty}\right| L^{\frac{1}{\nu}}$, showing the data collapse obtained by taking $\nu=1, \beta=0.125$ and $T_{c}^{\infty}=1.748$. If we fit the data, we recover $\beta$ from the slope of the $T>T_{c}^{\infty}$ branch (see full line). Lower panel: Plots of $U$ versus $T$ as obtained for samples of different size and for the same choice of $D$ and $\alpha$, showing the common intersection point at the critical temperature given by $T_{c}^{\infty} \simeq 1.748$, in agreement with the value used to obtain data collapse (upper panel). Figure obtained by using MU algorithm.

\section{First-Order Transitions and Tricritical Points}

Figure 3 shows an example of how the transition from the paramagnetic to the ferromagnetic state as a function of $D$ turns from first-order at low temperatures to secondorder as the temperature is raised. This is evidenced in the softening of the discontinuity in $M$ plotted in the figure for a fixed value of $\alpha$ and for a series of different temperatures.

To determine the first-order line and the position of the tricritical point we used the WL algorithm with the 


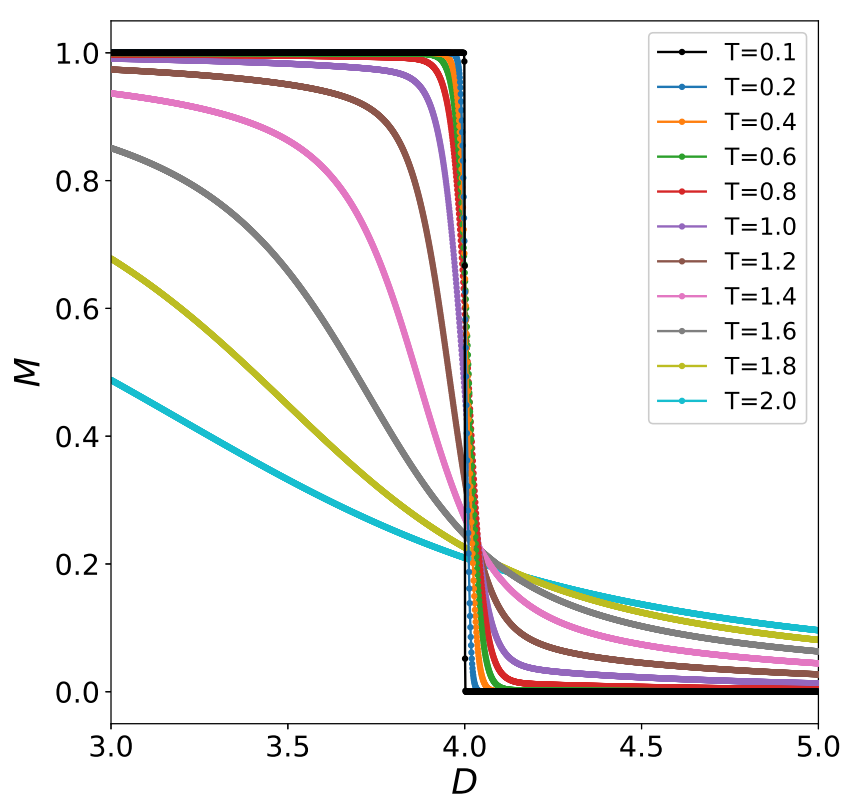

FIG. 3. Plot of the magnetisation $\langle|m|\rangle$ vs the crystal field $D$, for $L=16$ and $\alpha=1$. Figure obtained by using $\mathrm{W}-\mathrm{L}$ algorithm.

aid of an accurate method discussed by Wilding and Nielaba $^{22}$, which was subsequently successfully used by several authors ${ }^{20,23,40,41}$. In these works, the authors calculate the distribution $P(\mathcal{D})$ of a new order parameter defined as $\mathcal{D}=\mathcal{N}-s E$, where $s$ is a field mixing parameter which represents the asymmetry of the scaling fields at the transition point, and can depend on the exact location along the coexistence line and on the system size. Since we are in the vicinity of the tricritical point, we take as an Ansatz $s=0.5$, and tune the crystal field and the temperature in order to obtain a double peaked function with equal heights. This gives the location of the first-order transition, and from this procedure the corresponding line can be determined (and hence the finite size tricritical point, in which this line formally terminates).

One can observe in Fig. 4 that the two peaks are well split in the first-order transition region but if we move along the transition line, the peaks start to merge together, which implies that the character of the transition is changing. In this way, the finite size tricritical points can be determined by comparing the separation of the peaks when the lowest value of the probability is $0.5 \%$ of the highest. As shown in Fig. 1, the first-order region gets smaller as $\alpha$ tends to -1 , and when $\alpha \leq-1$ this region disappears.

Further check on the location of the tricritical point can be obtained by means of the use of the sizeindependent crossing point of the fourth-order cumulant calculated for the variable $P(\mathcal{D})$. First one estimates the tricritical crystal field, $D_{t c}$, from the extrapolation of the finite size tricritical points for lattices of different sizes as it is shown in Fig. 5, by using the tricritical

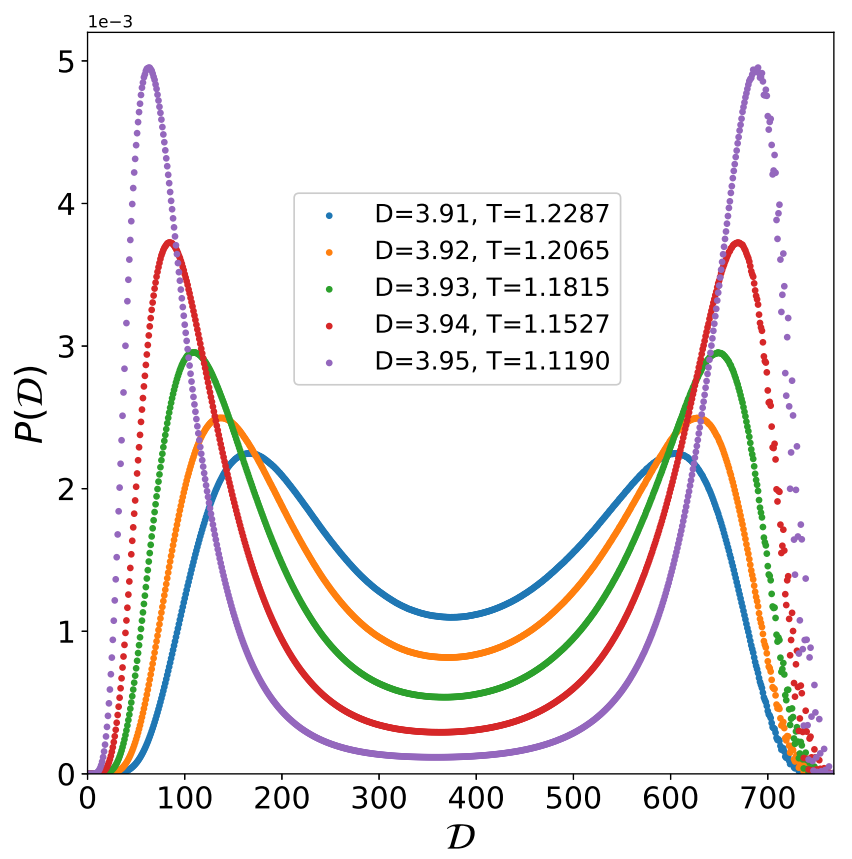

FIG. 4. Probability distribution function of $\mathcal{D}$ for $\alpha=1$ and $L=16$, showing the double peaks at the same heights for different values of $\mathrm{D}$ and $\mathrm{T}$. Figure obtained by using W-L algorithm.

exponents $^{42} \frac{1}{\nu}=1.86(2)$ and $\beta=0.0453(2)$. Then, the temperature can be obtained by the crossing point of the fourth-order cumulant, measured at $D_{t c}$. Our estimate of the tricritical point for $\alpha=0$ is $T_{t c}^{\infty}=0.6078(2)$ and $D_{t c}=1.96658(2)$. The error estimation is only graphical since the calculation is based on a single sample of the WL density of states. This result is in good agreement with the previous results for the spin-1 BlumeCapel model in square lattices, e.g, $T_{t c}^{\infty}=0.610(5)$ and $D_{t c}=1.965(5)^{43}, T_{t c}^{\infty}=0.608(1)$ and $D_{t c}=1.9665(3)^{22}$, $T_{t c}^{\infty}=0.609(4)$ and $D_{t c}=1.965(5)^{44}, T_{t c}^{\infty}=0.609(3)$ and $D_{t c}=1.966(2)^{21}$, and $T_{t c}^{\infty}=0.608(1)$ and $D_{t c}=$ $1.9665(3)^{23}$. We have also estimated the tricritical point for $\alpha=1, T_{t c}^{\infty}=1.1545$ and $D_{t c}=3.9342$ and $\alpha=2$, $T_{t c}^{\infty}=1.6679$ and $D_{t c}=5.9207$. The shift on the tricritical points caused by the lattice size is $<1 \%$.

Once we know the tricritical temperature, we can obtain the distribution probability function for different lattice sizes at $T_{t c}^{\infty}$. We use the normalized variable $\tilde{\mathcal{D}}=\mathcal{D}-\langle\mathcal{D}\rangle$ in order to have zero average and unit variance. Its distribution function can then be obtained from the finite-size scaling Ansatz ${ }^{22}$

$$
P_{L}(\tilde{\mathcal{D}})=L^{\left(d-\frac{1}{\nu}\right)} p_{\mathcal{D}}^{*} L^{\left(d-\frac{1}{\nu}\right)} \tilde{\mathcal{D}}
$$

where $p_{\mathcal{D}}^{*}$ is a universal function and $d$ is the dimension of the lattice, in this work $d=2$. As it is shown in the inset of Fig. 6, the probability distributions for different lattice sizes, with the tricritical exponent $\frac{1}{\nu}$, successfully collapse onto a single curve. The possible error in this 
estimate with the shape of the distributions, mainly originates from the discrete nature of $\mathcal{D}$, which affects the visualization of its histogram, specially for small systems, and thus can cause ambiguity in the determination of the mixing parameter.

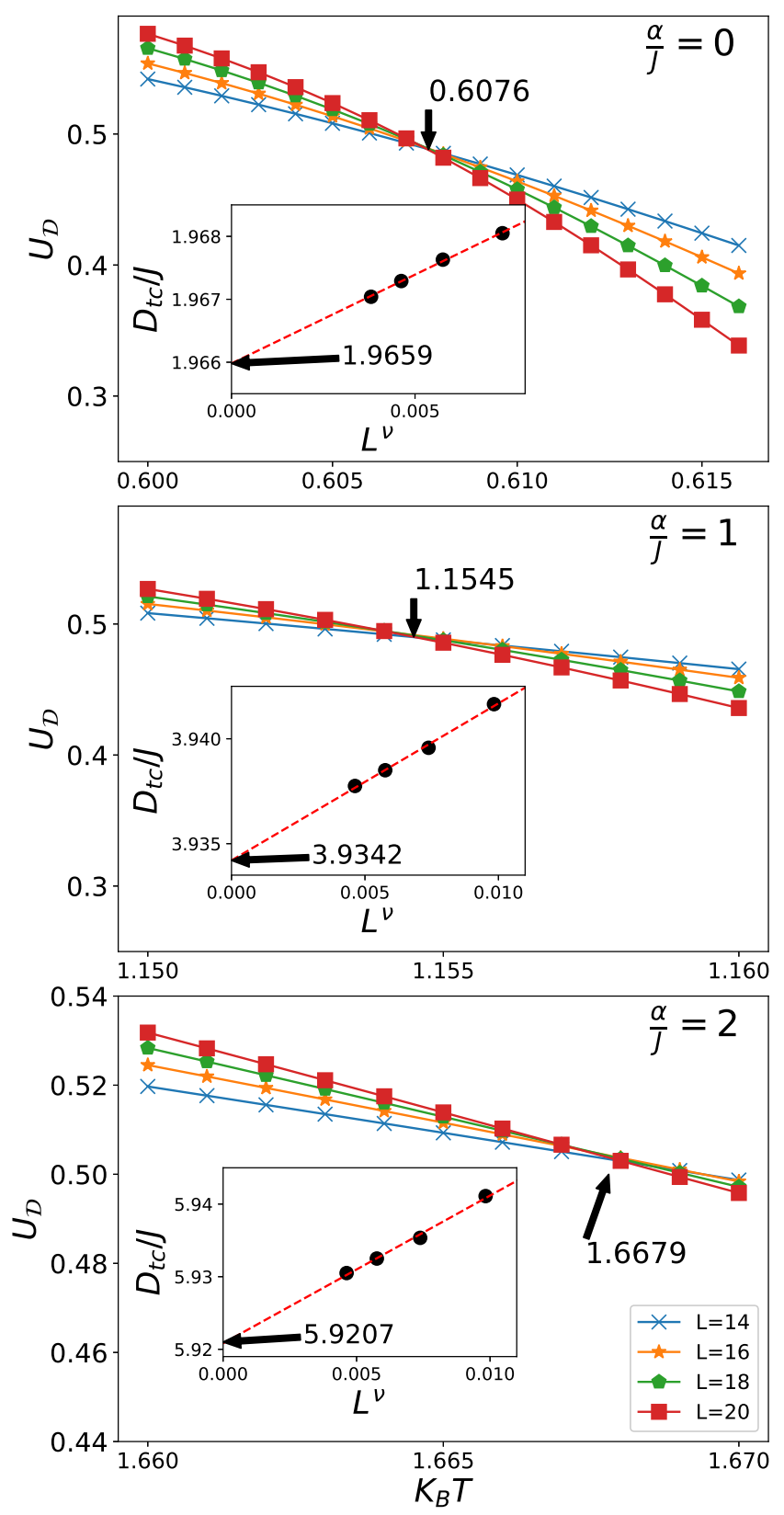

FIG. 5. Location of the tricritical point for $\alpha=0, \alpha=$ 1 and $\alpha=2$. The tricritical temperature is determined at the crossing point of the fourth-order cumulant, while the extrapolation of the transition points with $\nu=1.86$ provide an estimation of the tricritical crystal field (inset). The sizes of the lattices are 14, 16, 18, 20.Figure obtained by using W-L algorithm.

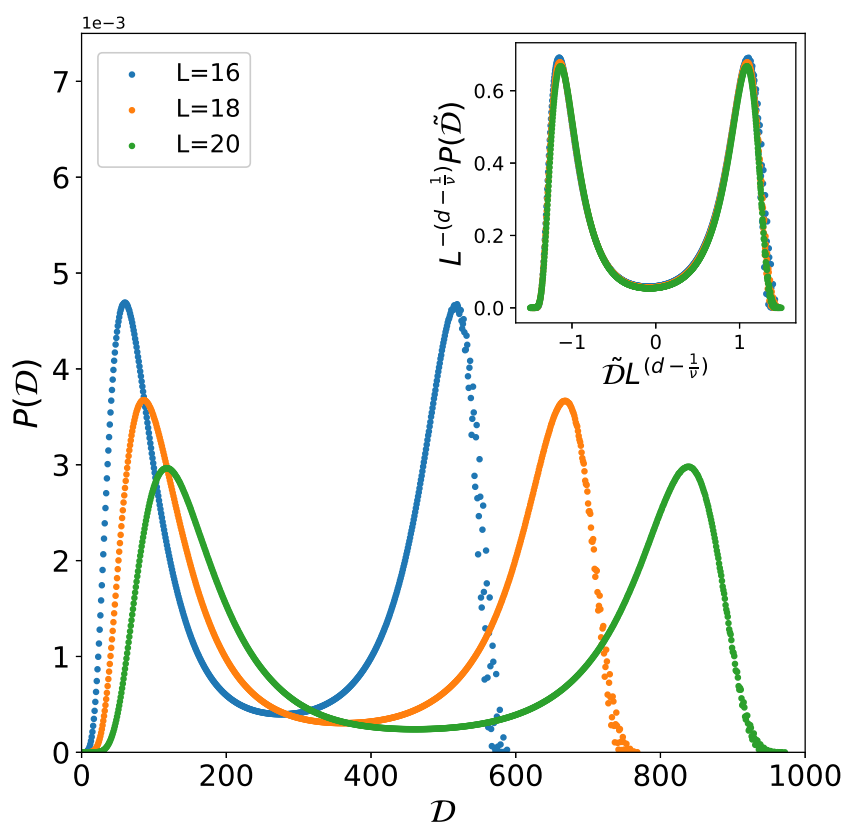

FIG. 6. Probability distribution functions of the variable $\mathcal{D}$ for $\alpha=1$, showing the double peaks for different lattice sizes at the tricritical point, $T_{t c}^{\infty}=1.1545$. The scaling plots of the probability distribution function are shown in the inset, with the tricritical exponent $\frac{1}{\nu}=1.86, d=2$ as we are working on a two dimensional lattice, and $\tilde{\mathcal{D}}$ is normalized to have zero average and unit variance. Figure obtained by using W-L algorithm.

\section{Quadrupolar Phase}

The analysis of the quadrupolar transition follows very similar lines. The main results were obtained by using W-L algorithm, but we perform a finite-size study using the MU algorithm to compare the accuracy of the method. The upper panel of Fig. 7 shows the order parameter $Q$ as a function of temperature for samples of different size (indicated in the legend) and for $\alpha=-5$ and $D=-8$. The inset shows the collapse of the data when the reduced variables $Q L^{\frac{\beta}{\nu}}$ and $\left|T-T_{c}^{\infty}\right| L^{\frac{1}{\nu}}$ are used. From this we checked that the universality class corresponds to Ising $2 \mathrm{D}$, i.e. $\nu=1, \beta=0.125$ and determined the critical temperature $T_{c}^{\infty}=2.75$. The lower panel shows the cumulant for $Q, U_{Q}$ as a function of temperature for samples of different size and the same choice of parameters. No first order transition is observed for $Q$.

Finally, to determine the thermodynamic limit of the critical temperature in the case of second order phase transitions we have performed finite size analysis of the finite size critical temperature. Fig. 8 shows a typical plot of size dependence of the finite size critical temperature $T_{c}(L)$ as determined from the position of the peaks of the susceptibility. Using the scaling relation

$$
T_{c}(L)=T_{c}^{\infty}+C L^{\frac{-1}{\nu}}
$$




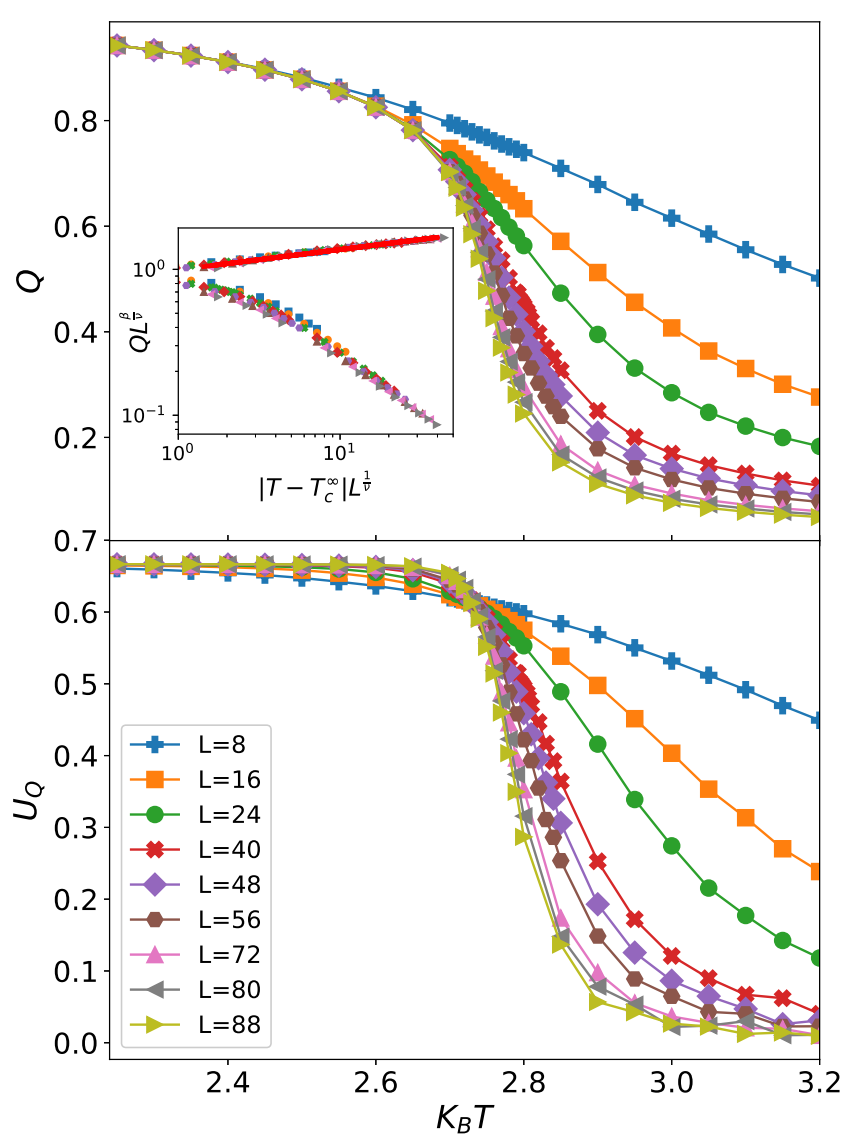

FIG. 7. Upper panel: plot of the quadrupolar moment versus temperature. The sizes of the lattice are shown, and the parameters are $\alpha=-5$ and $D=-8$. Inset: Scaling plot of the $Q L^{\frac{\beta}{\nu}}$ vs $\left|T-T_{c}^{\infty}\right| L^{\frac{1}{\nu}}$, showing the data collapse obtained by taking $\nu=1, \beta=0.125$ and $T_{c}^{\infty}=2.75$. If we fit the data, we recover $\beta$ from the slope of the $T>T_{c}^{\infty}$ branch (see full line). Lower panel: Plots of $U_{Q}$ versus $K_{B} T$ as obtained for samples of different size and for the same choice of $D$ and $\alpha$. Figure obtained by using MU algorithm.

where $T_{c}^{\infty}$ is the critical temperature in the thermodynamic limit, one can calculate $T_{c}^{\infty}$ as the extrapolation of the plot of $T_{c}(L)$ versus $L^{\frac{-1}{\nu}}$ (shown in the inset). The extrapolation yields $T_{c}^{\infty}=2.75$ for this particular choice of the parameters $(\alpha=-5$ and $D=-8)$, in excellent agreement with the results already shown in figure 5 . So, a combined simulation study based on WL and MU algorithms allow us to determine the full phase diagram of the BEG model (c.j. Fig. 1). Results obtained by using both approaches are fully consistent among them, as well as with exact calculations for the ground state.

\section{NEXT NEAREST NEIGHBORS INTERACTION}

The checkerboard arrangement of vacancies and occupied sites in the two disjoint sublattices of the square

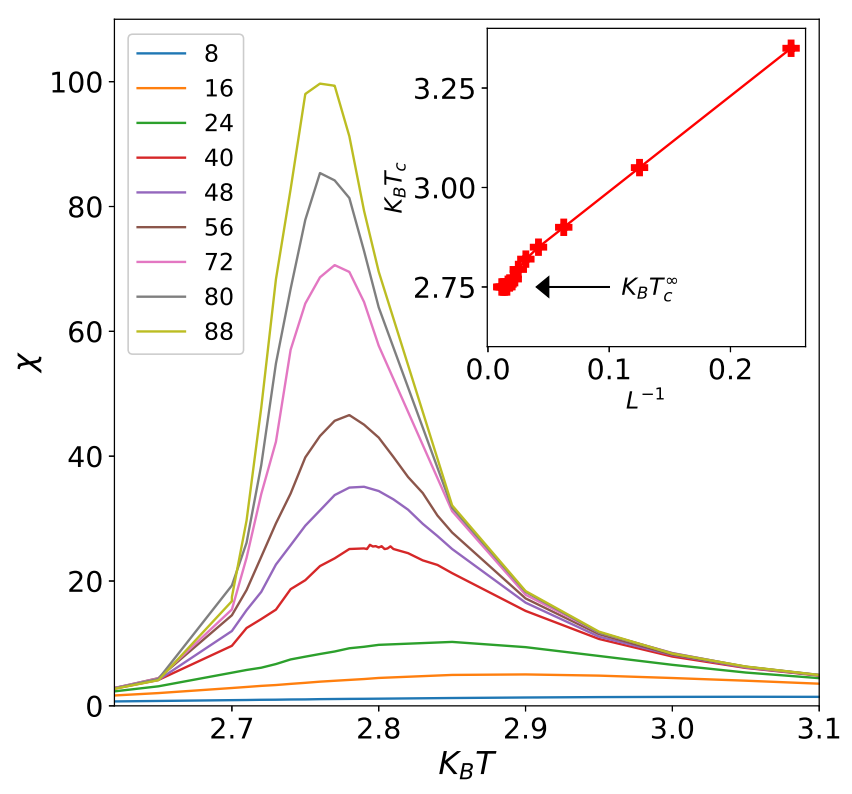

FIG. 8. Plot of the size dependence of the finite size critical temperature $\left(T_{c}(L)\right)$ as determined from the position of the peaks of the susceptibility, for this particular choice of the parameters $(\alpha=-5$ and $D=-8)$. Figure obtained by using MU algorithm.

lattice that happens at the quadrupolar phase, causes that the spins get segregated from each other by the vacancies. It is therefore of interest to study the behavior of the system by adding a next nearest neighbour (NNN) interaction to induce spin order in the quadrupolar phase. The NNN interaction is characterized by the coupling constant $J_{2}$ which in this instance we take to be of the same sign than the NN interaction. The Hamiltonian is given by:

$$
\mathcal{H}=\mathcal{H}_{\mathrm{BEG}}-J_{2} \sum_{\langle\langle i, j\rangle\rangle} s_{i} s_{j}
$$

where $\langle\langle i, j\rangle\rangle$ indicates the sum over the next nearest neighbors.

As in III A one can also calculate the $D-\alpha$ phase diagram at $T=0$. For $\alpha>-1$ one can still find the ferromagnetic phase which, depending on the number of vacancies, can lead to the paramagnetic phase, along the line given by

$$
D=2 J(\alpha+1)+2 J_{2} .
$$

For $\alpha<-1$ one recovers the quadrupolar phase that takes place along the line

$$
D=4 J(\alpha+1)+2 J_{2},
$$

but this time the sublattice filled with spins will show a ferromagnetic order for any non-zero $J_{2}$. We call $Q_{F}$ this phase where quadrupolar and ferromagnetic order coexist. Like in the NN case, even at $T=0$ the system 
will eventually disorder into a paramagnetic phase, but in this case it will happen above $D=2 J_{2}$. From this analysis and figure 9 , one can easily see that as $J_{2}$ increases, the ferromagnetically ordered phase grows over the disordered phase, while the quadrupolar phase maintains its size.

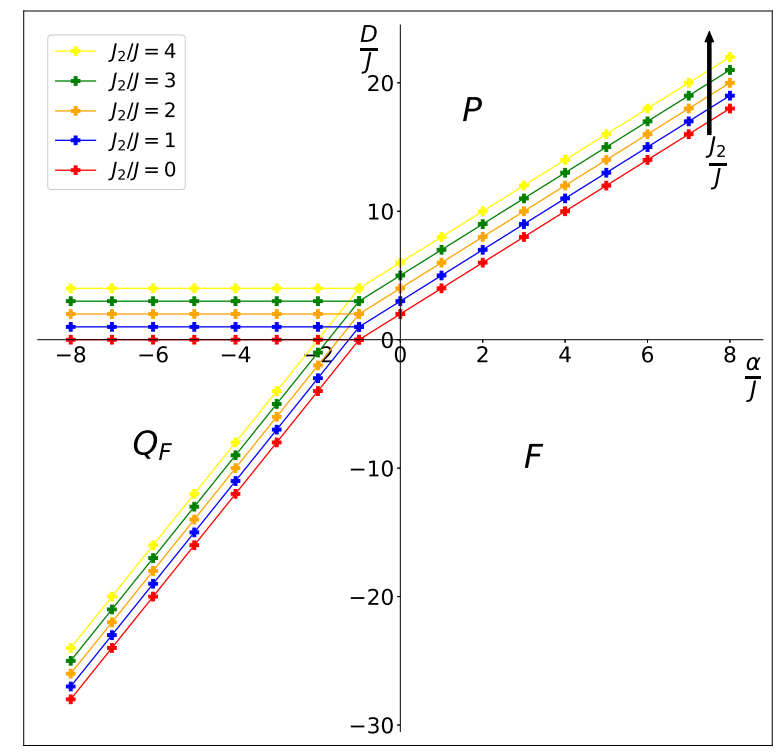

FIG. 9. Phase Diagram of the BEG Model with NNN interaction at $T=0$. It shows the behavior of the three phases: ferromagnetic $(F)$, paramagnetic $(P)$ and ferromagneticquadrupolar $\left(Q_{F}\right)$, as $J_{2}$ increases. When $J_{2}=0$ one recovers the BEG phase diagram for $T=0$ shown in figure 1 .

Figure 10 shows the phase diagram, obtained with W-L algorithm, as function of $D, J_{2}$ and $T$ when $\alpha=-5$. It is interesting to notice that as $J_{2}$ increases, the quadrupolar phase with ferromagnetic order, the $Q_{F}$ phase found at $T=0$, starts to develop at finite temperatures till above a given ratio of $J_{2} / J$, between 1 and 1.5 , all quadrupolar order shows ferromagnetism. The transition between the quadrupolar phase and the quadrupolar phase with ferromagnetic order is second order, as shown in Figure 11, and corresponds to the Ising 2D universality class. For large negative $D$ the order-disorder transition lines tend to the 2D Ising model with NNN interaction. The critical temperature we obtain matches the corresponding Ising temperatures which can be obtained by solving ${ }^{45}$

$$
2 e^{-\frac{2 J}{k_{B} T_{C}}}\left(1-e^{-\frac{2 J}{k_{B} T_{C}}}\right)-e^{-\frac{2\left(J+J_{2}\right)}{k_{B} T_{C}}}-e^{-\frac{4 J_{2}}{k_{B} T_{C}}}=0 .
$$

As we have mentioned before, the lattice size $L=16$ produces a shift on the critical temperature smaller than $6 \%$.

\section{CONCLUSIONS}

In this work we have performed a thorough analysis of the complete phase diagram of the BEG model. The pa-

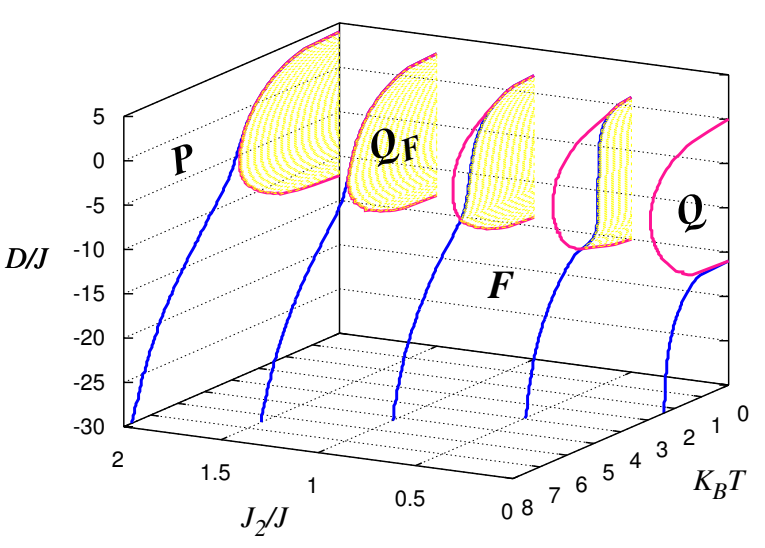

FIG. 10. Phase Diagram of the BEG Model with NNN interaction for $\alpha=-5$. It shows the behavior of the four phases: ferromagnetic $(F)$ which is under the blue line, paramagnetic $(P)$, outside the blue line, quadrupolar $(Q)$, inside the pink line and ferromagnetic-quadrupolar $\left(Q_{F}\right)$ the yellow region inside the pink line, as $J_{2}$ increases. When $J_{2}=0$ one recovers the BEG phase diagram shown in figure 1. Figure obtained by using W-L algorithm.

rameter $D$ controls the density of vacancies in the system, and it can drive it from the simple Ising limit $(D \rightarrow-\infty)$ where all sites are occupied with \pm 1 spins, to the case where the sparsity of interacting sites drives the system to disorder. The case of $\alpha=0$, the Blume-Capel model, shows a ferromagnetic transition that turns second to first order at a tricritical point. The inclusion of an effective interaction between vacancies, controlled by the parameter $\alpha$ leads to the emergence of the quadrupolar phase, with no magnetic order, but characterized by occupying a single sublattice of the square lattice. The transition to this phase is always second order. In this work we confirm the universality class of the transition to be $2 \mathrm{D}$ Ising, and determined the thermodynamic critical temperatures and the position of the tricritical line.

Based on studies of the BC model, it is well known that vacancies tends to segregate along the interface between domains with different orientations of the spins ${ }^{28-33}$. Since the BC model corresponds to the case $\alpha=0$, the study of the "enrichment of vacancies" at interfaces within the framework of the BEG model will be quite interesting, e.g. in the case of wetting phenomena. In fact, for the $\mathrm{BC}$ model the presence of vacancies at the interface may induce the occurrence of first-order and tricritical wetting behavior ${ }^{28-30}$. In this way we expect that by tuning the parameter that regulates the interaction among vacancies $\alpha$, the BEG will exhibit an interesting and rich wetting behaviour, which we are planning to study in the near future. In this sense, the knowledge and understanding of the phase diagram of the BEG model, as discussed in the present paper, is a prerequisite to 


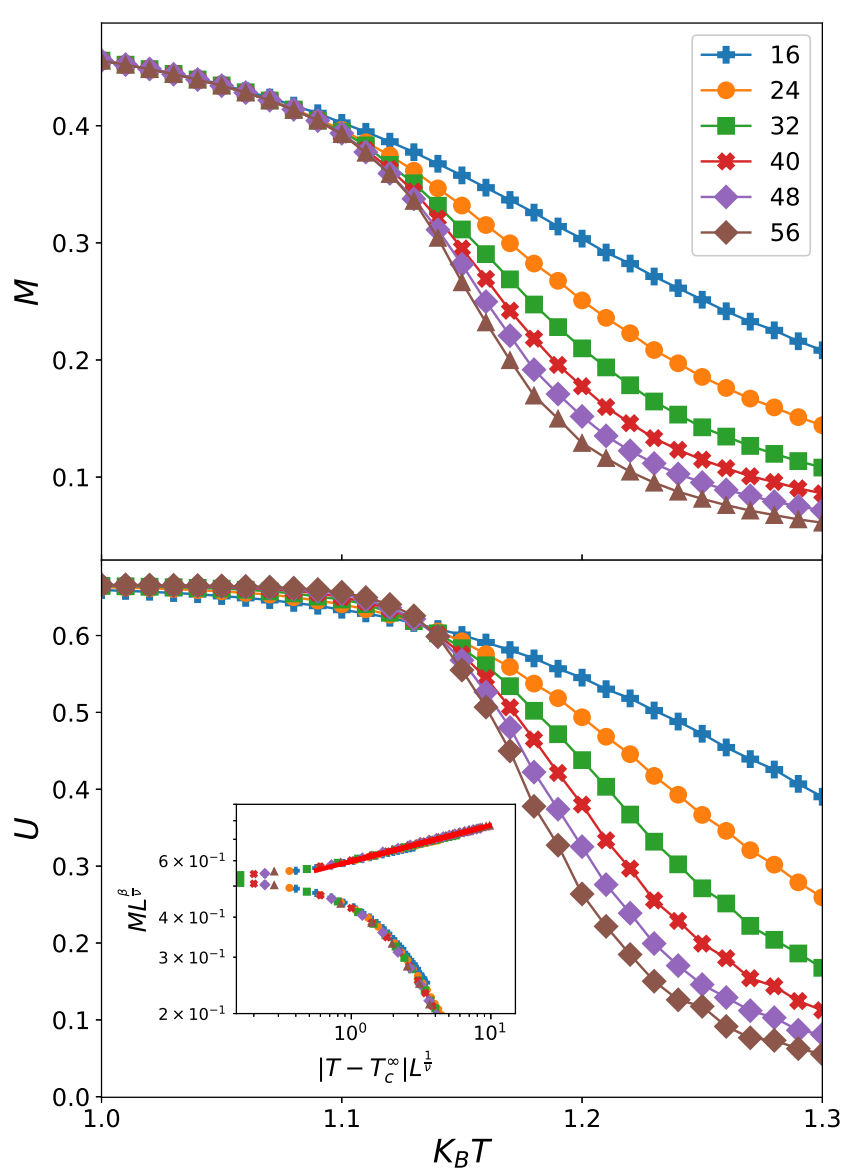

FIG. 11. Upper panel: plot of the magnetization $\langle|m|\rangle$ vs temperature. The sizes of the lattice are shown, and the parameters are $J_{2}=0.5, \alpha=-5$ and $D=-8$. Inset: Scaling plot of $M L^{\frac{\beta}{\nu}}$ vs $\left|T-T_{c}^{\infty}\right| L^{\frac{1}{\nu}}$, showing the data collapse obtained by taking $\nu=1, \beta=0.125$ and $T_{c}^{\infty}=1.13$. If we fit the data, we recover $\beta$ from the slope of the $T>T_{c}^{\infty}$ branch (see full line). Lower panel: Plots of $U$ versus $T$ as obtained for samples of different size and for the same choice of $D$ and $\alpha$, showing the common intersection point at the critical temperature given by $T_{c}^{\infty} \simeq 1.13$, in agreement with the value used to obtain data collapse (upper panel). Figure obtained by using MU algorithm. undertake further studies.
1 M. Blume, V. J. Emery, R. B. Griffiths; Phys. Rev. A 4(3), 1071, (1971).

2 M. Blume; Phys. Rev. 141, 517, (1966).

${ }^{3}$ H. W. Capel; Physica 32, 966, (1966).

4 D. Mukamel, M. Blume; Phys. Rev. A 10, 610, (1974).

5 D. Furman, S. Duttagupta, R. B. Griffiths; Phys. Rev. B 15, 441, (1977).

6 M. Schick, W. Shih; Phys. Rev. B 34, 1797, (1986).

7 K. E. Newman, J. D. Dow; Phys. Rev. B 27, 7495, (1983).

8 S. A. Kivelson, V. J. Emery, H. Q. Lin; Phys. Rev. B 42, 6523, (1990).

9 D. M. Saul, M. Wortis, D. Stauffer; Phys. Rev. B 9, 4964, (1974).
10 Yung-Li Wang, F. Lee, J. D. Kimel; Phys. Rev. B 36, 8945, (1987).

11 A. N. Berker, M. Wortis; Phys. Rev. B 14, 4946, (1976).

12 O. F. de Alcantara Bonfim, F. C. Sá Barreto; Phys. Lett. A 109, 341, (1985).

13 F. C. Sá Barreto; Rev. Bras. de Fisica 20, 152, (1990).

14 C. Buzano, L. Evangelista; J. Magn. Magn. Mat. 104, 231, (1992).

15 K. G. Chakraborty; J. Phys. C 21, 2911, (1988).

16 T. Kaneyoshi, E. F. Sarmento; Physica A 152, 343, (1988).

17 T. Kaneyoshi; Physica A 164, 730, (1990).

18 J. Zierenberg, N. G. Fytas, M. Weigel, W. Janke, A. Malakis; Eur. Phys. J. Special Topics 226, 789, (2017). 
19 J. Zierenberg, N. G. Fytas, W. Janke; Phys. Rev. E 91, 032126, (2015).

${ }^{20}$ W. Kwak, J. Jeong, J. Lee, D.-H. Kim; Phys. Rev. E. 92, 022134, (2015).

21 C. J. Silva, A. A. Caparica, J. A. Plascak; Phys. Rev. E 73, 036702, (2006).

22 N. B. Wilding, P. Nielaba; Phys. Rev. E 53, 926, (1996).

23 J. A. Plascak, P. H. L. Martins; Comput. Phys. Commun. 184, 259, (2013).

24 N. G. Fytas; Eur. Phys. J. B 79, 21, (2011).

25 A. Malakis, A. Nihat Berker, I. A. Hadjiagapiou, N. G. Fytas, T. Papakonstantinou; Phys. Rev. E 81, 041113, (2010).

26 A. Malakis, A. Nihat Berker, I. A. Hadjiagapiou, N. G. Fytas; Phys. Rev. E 79, 011125, (2009).

27 R. E. Belardinelli, V. D. Pereyra; Phys. Rev. E 75, 046701, (2007).

28 E. V. Albano, K. Binder; Phys. Rev. Lett. 109, 036101, (2012).

29 E. V. Albano, K. Binder; Phys. Rev. E 85, 061601, (2012).

30 M. L. Trobo, E. V. Albano, K. Binder; Phys. Rev. E 90, $022406,(2014)$.

31 W. Selke, J. Yeomans; J. Phys. A 16, 2789, (1983).

${ }^{32}$ W. Selke, D. A. Huse, D. M. Kroll; J. Phys. A 17, 3019, (1984).

33 W. Selke; Surf. Sci. 144, 176, (1984).

${ }^{34}$ P. M. Chaikin, T. C. Lubensky; Principles of Condensed Matter Physics (Cambridge University Press, Cambridge, UK), 179, (1995).

${ }^{35}$ D. P. Landau, K. Binder; A Guide to Monte Carlo Simulations in Statistical Physics, 4th ed. (Cambridge University Press, Cambridge, UK), (2015).

${ }^{36}$ N. Metropolis, A. W. Rosenbluth, M. N. Rosenbluth, A. M. Teller, E. Teller; J. Chem. Phys. 21, 1087, (1953).

37 F. Wang, D. P. Landau; Phys. Rev. Lett. 86, 2050, (2001).

38 F. Wang, D. P. Landau; Phys. Rev. E 64, 056101, (2001).

39 Y. I. Dublenych; Phys. Rev. B 71, 012411, (2005).

40 N. B. Wilding, A. D. Bruce; J. Phys. Condens. Matter 4, 3087, (1992).

41 A. D. Bruce, N. B. Wilding; Phys. Rev. Lett. 68, 193, (1992).

42 R. da Silva, N. A. Alves, J. R. Drugowich de Felicio; Phys. Rev. E 66, 026130, (2002).

43 P. D. Beale; Phys. Rev. B 33, 1717, (1986).

44 J. C. Xavier, F. C. Alcaraz, D. Pena Lara, J. A. Plascak; Phys. Rev. B 57, 11575, (1998).

45 H. J. W. Zandvliet; Europhys. Lett. 73, 747, (2006).

\section{Appendix: Numerical methods}

The Metropolis update (MU) is a Markov Chain Monte Carlo method that allows to obtain a sequence of random samples from a probability distribution. Simulations are started from a random configuration of spins and vacancies. Then the energy $E_{i}$ of this initial configuration is computed, and subsequently a new configuration, with energy $E_{j}$, is attempted just by flipping a randomly selected spin (or vacancy). The transition probability between those configurations, given by $\omega=\exp \left[\beta\left(E_{j}-E_{i}\right)\right]$; $\beta=1 / k_{B} T$ is evaluated, and the trial is accepted if $\omega>r$, where $r \in(0,1)$ is a pseudo random number and
$k_{B} T$ is the Boltzmann constant. A Monte Carlo time step (MCS) involves $N=L \times L$ flipping attempts, so that each spin of the system is visited once in average. This procedure is followed during $\tau_{D}=6 \times 10^{6} \mathrm{MCS}$ in order to allow for the equilibration of the system. Then, data is recorded during $\tau_{M}=16 \times 10^{6} \mathrm{MCS}$, in order to perform an average over the quantities of interest, such as magnetization, susceptibility, cumulant, etc.

Even though this is a very useful algorithm to study many particles systems, in the case of the BEG model it shows some disadvantages that are successfully solved by the Wang-Landau algorithm. Among others, we can quote that the MU algorithm works properly if all the configurations of the system are into a relatively narrow energy range, otherwise, there is the possibility that, during the random walk, the process gets stuck in an energy local minimum from where, the probability to escape is very low. Furthermore, the MU method only allows to set one temperature per run, so one has to perform many runs in order to get the data for many temperatures.

Unlike the usual MU algorithm that directly estimates the mean values of the thermodynamic observables at a given temperature, the Wang-Landau (WL) algorithm accurately estimates the density of states $g$ via a random walk. We work in the grand canonical ensemble, interpreting $D$ in the second term of the BEG Hamiltonian to be a chemical potential. The number of spins/particles is then given by $\mathcal{N}=\sum_{i=1}^{N} s_{i}^{2}$. The energy $E$ is given solely by the interaction terms: the first and third terms of eq. 1 for the NN case, and the first, third and fourth terms of eq. 9 for the NNN case. In order to compute the grandcanonical partition function and calculate the thermodynamic quantities under an applied magnetic field we seek to determine $g(E, \mathcal{N}, M)=1$, where $M=\sum_{i=1}^{N} s_{i}$ is the magnetization. The algorithm samples the configuration space by randomly flipping spins. New configurations of the system are accepted with a probability that is proportional to $1 / g(E, \mathcal{N}, M)$, the reciprocal of the density of states. As a result a flat histogram $H E(E, \mathcal{N}, M)$ is generated during the random walk and the current density of states is modified by a refinement parameter $f$. Since the density of states is not known a priori, in the beginning of the simulation one sets all entries to $g(E, \mathcal{N}, M)=1$ for every state and perform a random walk considering that, if $\left(E_{1}, \mathcal{N}_{1}, M_{1}\right)$ and $\left(E_{2}, \mathcal{N}_{2}, M_{2}\right)$ are the states before and after a spin is flipped, the transition probability would be

$$
\begin{array}{r}
p\left(\left(E_{1}, \mathcal{N}_{1}, M_{1}\right) \rightarrow\left(E_{2}, \mathcal{N}_{2}, M_{2}\right)\right)= \\
\min \left(\frac{g\left(E_{1}, \mathcal{N}_{1}, M_{1}\right)}{g\left(E_{2}, \mathcal{N}_{2}, M_{2}\right)}, 1\right),
\end{array}
$$

and the density of states is modified as

$$
g(E, \mathcal{N}, M) \rightarrow g(E, \mathcal{N}, M) f,
$$

When choosing the refinement parameter one should have in mind that all possible energy levels must be reached quickly even for a large system. A reasonable 
choice is $f=f_{0}=e$, and to reduce it one can use a function like $f_{n+1}=\sqrt{f_{n}}$. This reduction is accomplished whenever the histogram becomes flat during the random walk. In our simulations, the flatness criterion for the histogram is about $80 \%$ of the average histogram $\langle H E(E, \mathcal{N}, M)\rangle$ and is generally checked every $1000 \mathrm{MC}$ steps. Afterwards, the histogram is reset to 0 and the simulation comes to and end when the modification factor is smaller than $1 e^{-8}$. Convergence of large systems is always difficult in the WL algorithm, moreover, every time a new parameter is added to $g$ the convergence becomes much harder since it requires smoothness on a higher dimensional space. In our case we require smoothness on a 3 dimensional space, which limited the size of our simulations. We have used the modification proposed by Belardinelli and Pereyra (BP), which changes the refinement parameter to $1 / \tau$, where $\tau=j / \epsilon$ is the Monte
Carlo time, $j$ is the number of trial moves attempted and $\epsilon$ is the number of energy levels of the system. Starting from the same initial condition as the original $\mathrm{WL}$ algorithm, the modification factor is reduced as $1 / \tau$, instead of checking the flatness condition after the condition $f_{n} \leq 1 / t$. The final value of $f$ should be fixed from the beginning. One additional advantage of the BP implementation is that its faster convergence speed makes it unnecessary to partition the energy spectrum. Thus, the full energy range can be used in all simulations and artifacts close to first-order transitions characteristic to multi-range implementations are avoided.

We have estimated the error of our simulations by looking at the different particular cases where our model has an exact solution. In all cases the error of the simulated data is below the symbol size of the figures. 